\title{
The Stable Wave Packet and Uncertainty
}

\author{
Antony J. Bourdillon \\ UHRL, San Jose, USA \\ Email: bourdillona@sbcglobal.net \\ Received 29 August 2015; accepted 13 November 2015; published 16 November 2015 \\ Copyright (C) 2015 by author and Scientific Research Publishing Inc. \\ This work is licensed under the Creative Commons Attribution International License (CC BY). \\ http://creativecommons.org/licenses/by/4.0/ \\ (c) (1) Open Access
}

\begin{abstract}
The traveling wave group that is defined on conserved physical values is the vehicle of transmission for a unidirectional photon or free particle having a wide wave front. As a stable wave packet, it expresses internal periodicity combined with group localization. An uncertainty principle is precisely derived that differs from Heisenberg's. Also derived is the phase velocity beyond the horizon set by the speed of light. In this space occurs the reduction of the wave packet which is represented by comparing phase velocities in the direction of propagation with the transverse plane. The new description of the wave function for the stable free particle or antiparticle contains variables that were previously ignored. Deterministic physics must always appear probabilistic when hidden variables are bypassed. Secondary hidden variables always occur in measurement. The wave group turns out to be both uncertain and probabilistic. It is ubiquitous in physics and has many consequences.
\end{abstract}

\section{Keywords}

Stable Wave Packet, Traveling Wave Group, Quantum Mechanics, Wave Mechanics, Determinism, Localization, Coherence

\section{Introduction}

The traveling wave group that is defined on conserved physical quantities is a stable wave packet. In opposition to the Copenhagen interpretation of Heisenberg's Uncertainty Principle, Schrödinger suggested that a particle may be represented by a wave packet [1], though the packet had been generally supposed unstable [2]. The purpose of this paper is to outline what can be learned from the simplest of all packets. We start with Popper's propensity theory for the wave function [3]; and we are now able to consider new consequences by analyzing the stable wave packet: we find that it has unique properties; that it is the decay product of unstable wave packets; that it contains hidden variables; that its implementation has hidden consequences; that it gives a new perspective on chronic problems; and that it gives a clear and precise derivation for the Uncertainty Principle. 
Schrödinger's equation described a wave mechanical solution for the Hamiltonian equation, where the sum of kinetic and potential energies, $H=T+V$ was conserved. The first and chief application for the equation is atomic physics. Working at the same time, Heisenberg developed a representation based on commuting operators. In particular he showed that any operator that commuted with the Hamiltonian operator was a constant of motion. The Uncertainty Principle came to him in a blinding flash [1] and was adopted by Bohr. Many scientists came to believe that "a problem had been previously resolved which they did not care too much about" [4] [5]. To seek the context for these developments, we have to refer back to Maxwell's electromagnetism and to Planck's law that described quantization in the energy of the photon in black body radiation.

In Maxwell's electromagnetism, the energy of a wave had depended on the intensities of its component electric field $\boldsymbol{E}$ and magnetic field $\boldsymbol{M}^{1}$. Planck's subsequent discovery showed that its energy depended on its frequency of oscillation. The wave packet shape and amplitude were intimately constrained and quantized. Thereafter, the wave intensities could be treated as secondary to the quantized energy that determined them. The further discovery of Bohr orbits in the atom showed that the de Broglie wavelength was likewise constrained. Meanwhile, the wave amplitude, developed from photons to particles, became a normalized function expressing uncertainty in position or in time. The extent of a wave packet, in time or space, was newly described by Heisenberg's Uncertainty Principle. Analytical methods were adapted for bosons and fermions. Previously, measurement had always contained a probabilistic foundation due to uncontrolled secondary aspects of measurement, even when physical laws had been deterministic, [6], (i.e. each event having only one possible outcome); but the new mechanics measured new kinds of object. They are vector states contained in complex Hilbert space, where measured variables help determine superpositions of elements in those states. The new type of quantized measurement, by hiding physical features evident in Maxwell's electromagnetism, posed a new issue for determinism, sometimes referred to as complementarity or as the wave-particle duality.

To address complementarity anew, in the light of the stable wavepacket, an initial statement is needed about suppositions that have been thoroughly discussed in the literature and that are accepted for the present purpose:

1) Realism: the world existed before my short life started, will continue after it is ended, and will do so whether I observe it or not.

2) Theory: scientists are free to invent any theory that explains physical phenomena or that simplifies a prior explanation. A theory must be discarded when falsified [7].

3) Complementarity was the term used by Bohr to describe the two aspects of particle behavior that is both corpuscular and wave-like. The former corresponds to our wave group envelope; the latter to its carrier wave, including interference and superposition. We shall see that in an event, the larger the uncertainty in momentum component the shorter is the wave packet in space; while the larger the uncertainty in energy, the smaller is the time taken by the wave packet to pass. The stable wave packet adds precision by showing how momentum and locality are complementary; as are energy and time.

4) Measurement: elimination of observer bias has always been a core concern in scientific measurement, and is not new in quantum mechanics. In particular it has always been the case that a measurement can alter probable outcomes in subsequent measurements. An example, in quantum theory, is the impossibility of measuring simultaneously two observables represented by non-commuting variables. However measurement in quantum mechanics was new because quantization required measurements of superpositions composed from elements of state vectors. Measurement required identification of those state vectors and determination of the relative coefficients (occurrence) of their elements.

5) Statistical interpretation: The wave function, which is considered below in further detail, has been understood in various ways. Among them are:

a) Random variations in measurements of determined properties due to uncontrollable experimental features. Typically results follow a normal distribution whose mean is the most probable value to be measured. This distribution implies hidden variables that are less significant than the quantity measured. For a sufficiently large sample, the accuracy of the mean depends on the square root of the number of measurements made, and this is not represented by the Uncertainty Principle described below. The Principle applies to single measurements and is derived, as below, from the wave function.

b) In statistical mechanics, probabilities are randomized, for example the number of atoms in a given volume

${ }^{1}$ In electromagnetism, $\boldsymbol{E}$ and $\boldsymbol{M}$ are both real, but they can be represented by a single complex exponential function that expresses their relative phase relationships by separating mathematically real and imaginary parts. The complex exponential conserves energy in the photon over a period of oscillation, as it does in other free bosons and fermions, where the imaginary components are not real. 
element, because practical measurements cannot give the locations and velocities of each atom. The statistical interpretation supposes that with full knowledge, the wave function would give way to deterministic corpuscular theory.

c) Even so, some values, including gas pressure, result directly from atomic collision due to randomized motion. They are therefore independent of knowledge deprivation.

6) Action at a distance: Though the well known thought experiment due to Einstein, Podolski and Rosen, [8] [9], was described to demonstrate incompleteness, it also treats localization. Given two particles A and B, originally in the same state, can a measurement on $\mathrm{A}$ at a later time give instantaneous information about the state of $\mathrm{B}$ ? If, at the time of measurement, the separation is sufficiently large, the information would contradict a tenet of special relativity. Bohr argued against any information without measurement; Einstein believed the information is localized. Experiments support localization [10]-[16], so does the stable wave packet.

7) Propensity is the theory for the wave function expounded by Popper [1]. It is agreed that its Hermitian product gives the probability for finding a particle or photon. What then is the wave function? Popper compared it to Aristotle's potential as a power to exist at a certain place or time. Propensity is the basis for the stable, meanvalued, wave packet.

8) Completeness claims under the Copenhagen interpretation [17] — continued even after the discovery of the positron, the neutron, the neutrino, and clusters of bosons, hadrons, leptons, quarks, and now even the Higg's boson-are surely dubious [1].

9) Quantum mechanics: Measurements determine coefficients for linear superpositions of elements of state vectors in complex Hilbert space. A wave function describes those states, typically through the vector that determines their coefficients. It can also be expressed in canonical variables by summation of elements multiplied by their coefficients. The elements may be found as solutions to state equations as classical analogues, or as observables determined by commutation relations given by the rules of quantum mechanics. The elements are quantized, either through constraints inherent in standing waves, or through other quantal properties.

The stable wave packet that is discussed here is wave mechanical. It contains constants of motion. We begin by using the packet to understand Uncertainty. With the consequent knowledge, further anomalies lead to further conclusions [18].

\section{The Stable, Mean-Valued, Wavepacket}

Dirac wrote [2] that the wave packet is unstable, but failed to note that without the packet there is no wave. The traveling wave group for a free particle or photon:

$$
\phi(\bar{k}, x, \bar{\omega}, t)=A \cdot \exp \left(\frac{X^{2}}{2 \sigma^{2}}+X\right) \text {, with } X=i(\bar{k} x-\bar{\omega} t),
$$

defined on values of mean wave vector $\bar{k}$ and mean angular frequency $\bar{\omega}$-is a stable wave packet on a wide transverse wave. Those two variables are themselves stable not only because they are mean values in a symmetric wave function; but their stability is guaranteed by respective conservation of momentum and energy; and triple guaranteed by symmetry in space-time. In one spatial dimension for simplicity, $X$ is an imaginary variable that causes $\phi$ to oscillate. If the real part represents the electric field in an electromagnetic wave, the imaginary part represents the relative phase of its (real) magnetic force field. The denominator $\sigma$ is particular because it depends on initial conditions, but it is stable during propagation in free space as a consequence of Newton's first law of motion. The variable determines the width of the wave packet, in either space or time, and can be defined as coherence. The normalizing amplitude $A$ depends on $\sigma$ and has corresponding stability. The envelope, $\exp \left(X^{2} / 2 \sigma^{2}\right)$, depends on the square of imaginary $X$, itself a function of four variables. Two are already described, so we are left with the variables $x$ and $t$ that describe the profile. Since the other variables are all stable, this profile is also stable. With a stable wave group, we can progress to new physics. The one dimensional equation is extensible to four space-time dimensions by appropriate substitution of the scalar product $\boldsymbol{k} \cdot \boldsymbol{r}$ for $k x$. Exceptional about this wave function is the fact that it is defined by mean values while implying a continuous range of component energies and momenta that are accessible by Fourier transforms. Back transforms on the Gaussian wave packet prove it stable. Moreover, beyond the plane wave approximation, edge effects are minimized as illustrated below. In Equation (1) and henceforth we use simplified units for the reduced Planck constant and for the speed of light $\hbar=c=1$. The stable wave packet is a solution to the wave equation [18]. 
To express the variables $\omega$ and $k$ implied in the equation, find the Fourier transform $F$ for the wave group. Write the probability:

$$
P(\omega)_{x=0}=F\left(\phi^{*}(t) \cdot \phi(t)\right)
$$

ignoring the constant normalizing amplitude $\mathrm{A}$, and evaluate:

$$
P(\omega)_{x=0}=\frac{\sigma}{\bar{\omega} \sqrt{2}} \exp \left(-\frac{\omega^{2} \sigma^{2}}{4 \bar{\omega}^{2}}\right) .
$$

Then proceed to write the uncertainty in angular frequency and energy as double the root of the argument to the exponential function in Equation (3), at the point where the probability falls to half maximum.

$$
\Delta \omega=4 \times 0.832 \frac{\bar{\omega}}{\sigma} .
$$

The Fourier back transform of Equation (3) gives the expected $\phi^{*} \phi$. Similarly

$$
P(t)_{x=0}=\exp \left(-\frac{t^{2} \bar{\omega}^{2}}{\sigma^{2}}\right) .
$$

Then, using the same criterion as for Equation (4),

$$
\Delta t=2 \times 0.832 \frac{\sigma}{\bar{\omega}} .
$$

Equations (4) and (6) yield

$$
\Delta \omega \cdot \Delta t=5.538=2 \pi \cdot 0.882
$$

and, translating to energy $E$ :

$$
\Delta E \cdot \Delta t=0.882 h
$$

Since $\hbar \cdot \Delta \omega=\Delta E$ when the unit of energy is generalized by the reduced Planck constant. Equation (8) is constant within the one-dimensional stable wave packet described, and is more precise than Heisenberg's value. Similar expressions can be found for $P(k)_{t=0}$ etc., and for the momentum, component $p_{x}$ :

$$
\Delta p_{x} \cdot \Delta x=0.882 h
$$

When the wave packet is not symmetric, the number may be larger, as in Heisenberg's equation; but the wave decays typically towards the stable packet as shown below in Section 3.3. The new derivation of the Uncertainty Principle in the free particle makes it a consequence of more fundamental wave mechanics. The derivation is not only well defined; it is also mathematically precise to any number of figures. Beyond the free particle, bound states are quantized by standing wave interference as in the Bohr atom, the Schrödinger equation, wave mechanics etc. Those are the states that quantize the photon. The precise result and clear definitions in Equations (8) and (9) raise several questions that we will come to after some minor illustrative observations. For the stable wave packet, the equality sign holds where Heisenberg's Principle has a limiting valuation. The well-defined product for the unidirectional wave is about 6 times greater than values given by Heisenberg for three dimensional systems.

In the simple one-dimension case, normalization gives $A_{x}=\overline{k_{x}} / \sqrt{2 \pi \sigma_{x}}$, leading, in three dimensions, to $A=\sqrt{\sum_{i} A_{i}^{2}}$ where $i=x, y, z$. Excepting the plane wave, $\sigma_{i}$ is often three dimensional. A depends on $\sigma$, since $\left\langle\phi^{*}, \phi\right\rangle=A^{*} A \cdot \sigma \sqrt{\pi}=1$, so that, in one dimension, $A=\sigma^{-1 / 2} \pi^{-1 / 4}$. In Bohr's quantum mechanics $A$ and $\sigma$ are suppressed together because they depend on the single quantized energy $\bar{\omega}$ as they do on $\bar{k}$, through $X$. The coherence, $\sigma$ is in principle observable and can be measured. It depends on initial conditions which may not form the Gaussian. If it were quantized, as by an initial emitting atom, $\sigma$ could not decay and the packet would be perfectly stable. Experimental data described below in Section 3.3 show ensembles decaying.

Meanwhile the expected value for the packet energy is derived from the gradient with respect to time, $-i \partial \phi / \partial t=\left(1+X / \sigma^{2}\right)(-i \bar{\omega}) \phi$. Since $\left.X\right|_{x=0}$ is a first order function of time, the integral on this symmetric group 
envelope vanishes leaving $\langle\omega\rangle=\bar{\omega}$. Similarly $\langle k\rangle=\bar{k}$, as expected. Operations on the Gaussian are comparatively easy.

The ideal stable wave packet is unidirectional, with a wide transverse field. However the theoretically stable Gaussian wave packet is not the only wave packet that is possible. Initial conditions determine a particular wave packet, but many examples in Fresnel diffraction [19] show that unstable packets rapidly lose their shapes because of edge effects. These cause decay to the relatively stable Gaussian, as will be discussed further in Section 3.3. When the Gaussian wave packet broadens in extent by an increase in $\sigma$, its momentum becomes more certain according to the equality sign. This may not be the case in unstable wave packets that supposedly hold to Heisenberg's limitation. The transverse momentum becomes uncertain when a packet passes through a transverse restriction in its path such as a slit.

Notice moreover that Equations (2)-(9) are consistent with the application of arbitrary realism. Suppose that a constant phase $\Phi$ is added to Equation (1) by initial conditions:

$$
\phi(\bar{k}, x, \bar{\omega}, t)=A \cdot \exp \left(\frac{X^{2}}{2 \sigma^{2}}+X+i \Phi\right) .
$$

The phase $\Phi$ expresses the relative displacement of phase in the carrier wave from the peak amplitude of the group (Figure 1). In Equation (2) it disappears, but might have an effect elsewhere as an example of a hidden variable. In Fresnel diffraction through a slit, the phase is known to lag [19]. In quantum mechanics, the phase is often included as a complex constant $c_{i}$ that includes an exponential function, $\exp (i \Phi)$, for example in the linear superposition of states $A$ and $B:|R\rangle=c_{1}|A\rangle+c_{2}|B\rangle$. We leave for another time the superposition of two states having different coherence.

These minor observations on the stable wave packet raise some general questions. The fundamental postulates of quantum mechanics are not affected: the linear superposition of states in complex Hilbert spaces; quantization of standing waves; commutation relations between observables and operators; measurement of states that are superposed but independent in orthogonal sets, etc. The questions we raise are as follows:

1) The Uncertainty Principle lacks precision and definition. Can it be improved through the example of the simple and stable wave packet, and through the application of wave mechanics?

2) The packet introduces new variables. What new information do they provide?

3) Can the new information alter probability measurements to make them more deterministic?

4) How, physically, are free particles quantized?

\section{Probability vs Determinism}

Any deterministic system containing an unknown hidden variable will appear probabilistic. Nevertheless, though

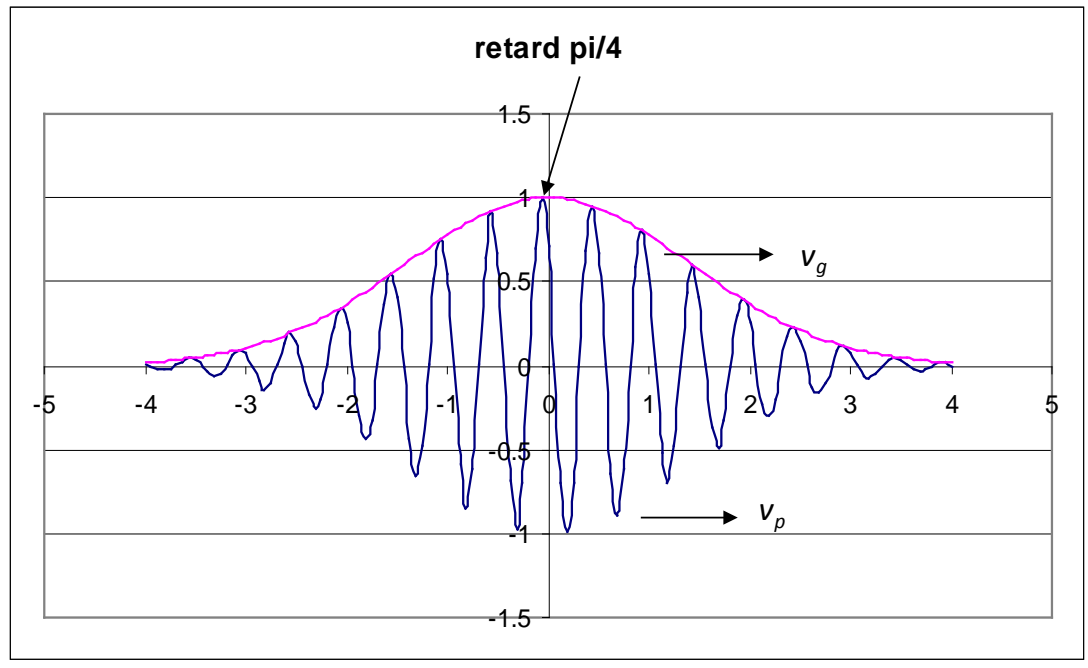

Figure 1. Real part of phase carrier wave (black), retarded by phase $\Phi=\pi / 4$ with respect to its wave group (red). The group velocity is $v_{g}$; the carrier velocity $v_{p}=c^{2} / v_{g}$. 
probability theory has always held a part in measurement (Section I.5a above), the method of physics from earliest times has been to isolate single variables and measure them as determined quantities. This requires elimination of hidden variables wherever they might be significant and where they can be eliminated. Meanwhile, a sufficient condition for a new variable to ensure determinism is that there is one-to-one correspondence between each value of the variable and each experimental outcome. This correspondence is still lacking.

\subsection{Probabilism in the Transverse Plane}

The stable wave packet that has been discussed so far is defined in the direction of propagation. Then the transverse plane is unrestricted. There are important features in both cases: unrestricted and restricted.

\subsubsection{The Unrestricted Transverse Plane}

In the direction of propagation, motion is subject to all the restrictions of relativity, for example, in particle kinetics with group velocity $v_{g}<c$; in electrodynamics; in solutions to the relativistic Klein-Gordon equation, etc. Operation of the last equation on Equation (1) yields, as output for a particle in free space without magnetic fields, an algebraic equation in second order:

$$
\hbar^{2} \omega^{2}=\hbar^{2} k^{2} c^{2}+m_{0}^{2} c^{4} .
$$

This is the same as is obtained from Einstein's well known relativistic formula by substituting for energy $E=\hbar \omega$ and for momentum $p=\hbar k$. The equation can be simplified with appropriate units $c=\hbar=1$. Differentiation then gives a new result in relativity, for the product of group velocity ${ }^{2} v_{g}$ and phase velocity $v_{p}$ :

$$
\frac{\mathrm{d} \omega}{\mathrm{d} k} \cdot \frac{\omega}{k}=v_{g} \cdot v_{p}=1 .
$$

In the line of propagation the group velocity is regular and relativistic; on the transverse plane, the group velocity approximately zero, and the carrier phase velocity, $v_{p}=c^{2} / v_{g}$, tends to infinity. The phase velocity describes wave motion beyond the event horizon of $c$. The speed of light functions as the Schwarzschild radius in cosmology, because the faster carrier wave is elastic, does not transport energy, and is not measured in special relativity. Though not directly measured in the conventional way, we know what it is: the inverse of the group velocity which is observable, and also the ratio of energy to momentum, each of which is an observable constant of motion in the free particle. When phase velocity beyond $c$ is accepted, multiple consequences follow. Its reference frame is the same as applies to the group velocity as described in special relativity for real space.

Firstly, for the plane wave, time in the transverse plane is Newtonian. Secondly, phase velocity provides a new concept for probability or propensity: propensity results from the instantaneous addition of wave amplitudes in the transverse plane. This explanation overcomes problems in a supra-relativistic spread of phase information when, at measurement, the wave packet is reduced: an example is the application of Fresnel integrals in diffraction; another is Bohr's concept of wholeness, which receives new life, though only within the coherence of the wave group. Thirdly, for antiparticles, a new set of dynamics is found as solutions to fundamental equations. The set is introduced through prior anomalies. Since the stable wave packet has been overlooked for so long, there should be no surprises:

a) Dirac did not think his calculation for the velocity of the electron in relativistic theory was anomalous. His application of Heisenberg's commutator formalism gives a value, for its velocity equal to $c$ [20], which is indeed a constant of the motion. This is the geometric mean of the group and phase velocities and represents an instantaneous wobble. However this observation of the geometric mean becomes acceptable only after space beyond the c-horizon is acknowledged. The true speed, as measured in vacuum tubes, is given, as a classical analogue, by the group velocity of the stable wave packet; the wobble is a theoretic curiosity. Notice that part of his wobble must be faster than $c$.

b) More seriously, Dirac discovered the negative energy solution for antiparticles but insisted that the mass is positive. The anomaly is arithmetical: at rest, a positive $m_{0} c^{2}$ cannot equal a negative $E$. His analogy of hole states in semiconductor valence bands makes problems for gravitation that we attempt to avoid.

c) Likewise he insisted that the kinetic energy of the antiparticle is positive. This later raised a problem for Feynman and Stueckelberg, whose principle states, “An antiparticle traveling forwarding time doesn't exist”

${ }^{2}$ Since $\overline{\overline{ }}$ and $\bar{k}$ are stable, the differential $\mathrm{d} \bar{\varpi} / \mathrm{d} \bar{k}$ has no value, but $\mathrm{d} \omega / \mathrm{d} k$ follows the relativistic constraint in Equation (11) and determines the group velocity. 
[21]-[23] $]^{3}$. However, another consequence occurs for the $c$-horizon: as $k \rightarrow m_{0}, \quad v_{g} \rightarrow \infty$ and $v_{p} \rightarrow 0$. The former result contradicts a tenet of relativity; neither result has been reported in experiments. These anomalies all disappear when, for the antiparticle, both $m_{0}$ and $k$ are negative.

Further consequences occur in other areas of physics: entanglement at a distance (since the wave packet is localized by its coherence); the wave function of a particle-antiparticle pair and their possible application to superconductivity, etc. However a more fundamental concern is the core of the argument for probabilism in quantum mechanics. This is normally illustrated by diffraction phenomena [1], so we shall consider what effect a new hidden variable might have on those phenomena. Moreover diffraction theory has significant details that we shall also consider.

\subsubsection{Diffraction in the Restricted Transverse Plane}

Following Newton, light was rectilinear, and more strictly now, relativistically geodesic, though locally Cartesian. This propagation provides the deterministic method of ray tracing that is applied, by approximation, in both light optics and in electron optics. The light bends sharply at a dielectric interface. When light passes through a narrow slit it diffracts so that some rays are observed to bend outside the aperture provided by the slit. The phenomena were consistently analyzed by Fresnel, using wave theory [19]. In particles, the same diffraction occurs, where the phenomena are analyzed in the same way after adjusting for corresponding wavelengths. The phenomenon is commonly used to demonstrate probabilism in physics. Beyond the near field, the illumination on a far screen will show an intensity distribution described, at least approximately, by a Gaussian. The bell shaped curve that may be measured is a probability distribution due to the product of the wave function with its complex conjugate at a given diffraction plane. It represents scattering of an ensemble. Suppose the intensity of the light beam is so reduced that light strikes the slit, one photon at a time. In the mechanics of Schrödinger or Heisenberg or Fresnel, there is no way of predicting beforehand, what path the light will take, nor where it will strike the screen on a selected diffraction plane.

\subsection{Flexible Ray Tracing in Fresnel Diffraction}

The straight line ray tracing that represents rectilinear theory must be made flexible to accommodate the wave theory. A method can be adapted to the Fresnel diffraction simulated in Figure 2. This shows scattering due to the emergence of a plane wave from a narrow horizontal slit. A cut of the calculated intensity on the plane at the Critical Condition [24] [25] is shown in the inset. At this condition ${ }^{4}$ the slit is optimally focused by Fresnel diffraction for high resolution proximity printing. There are two points to notice about these simulations. Firstly the progression of the diffraction is illustrated successively from plane to plane. Knowing that intensity is conserved, it is possible to construct flexing rays that follow the light transmission from the slit to a given diffraction plane. Secondly, we may ask again a question about the ray path taken by a particular photon. Suppose that the incident beam intensity is reduced until light is emitted and absorbed one photon at a time. When the photon is detected at a point on the diffraction plane, can we say which ray it followed, and can we predict the ray path in the future? The answer has to be, "No" to both questions for the following reason. The experimentally verified [26] probability profile shown in the inset is calculated from all rays emerging from the slit; not just one of them. The determination of where, on the diffraction plane, the photon is detected is not determined by the variables known in the wave propagation; but supposedly includes both emitter and absorber states and phases. Inclusion of all variables is, for sound experimental reasons, bypassed in the route taken by probabilism.

Notice the example that is given in the figure, of the decay of the transverse rectangular wave packet from the slit, to a Gaussian in far field. The decay to the Gaussian, owing to edge effects, is typical in Fresnel diffraction. The eventual divergence is itself an example of the Uncertainty Principle in the unstable wave packet in two spatial dimensions. A minimal prediction due to the Heisenberg Uncertainty Principle is represented by the dashed line in Figure 2. Before the slit, the horizontal momentum $p_{y} \simeq 0$. At the slit, $p_{y}$ depends on the Principle: $p_{y}=\Delta p_{y} \geq \hbar / w$. So the minimal uncertainty scattering angle: $q=p_{y} / p_{x}=(\hbar / w) /(h / \lambda)=\lambda /(2 \pi w)$, where $p_{x}$ is given by the de Broglie wavelength. The dashed line in the figure shows the prediction for increasing Uncertainty displacement, with downward passage, owing to the required minimal transverse momentum.

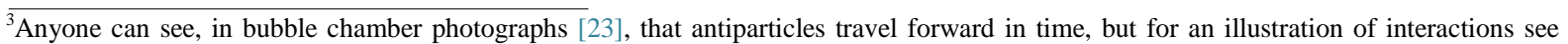
[18].

${ }^{4}$ In proximity X-ray Lithography, the condition is governed by wavelength, mask feature size and mask-wafer gap. That scattering amplitude is greatest for which the vector joins the extremities of Cornu's spiral.
} 


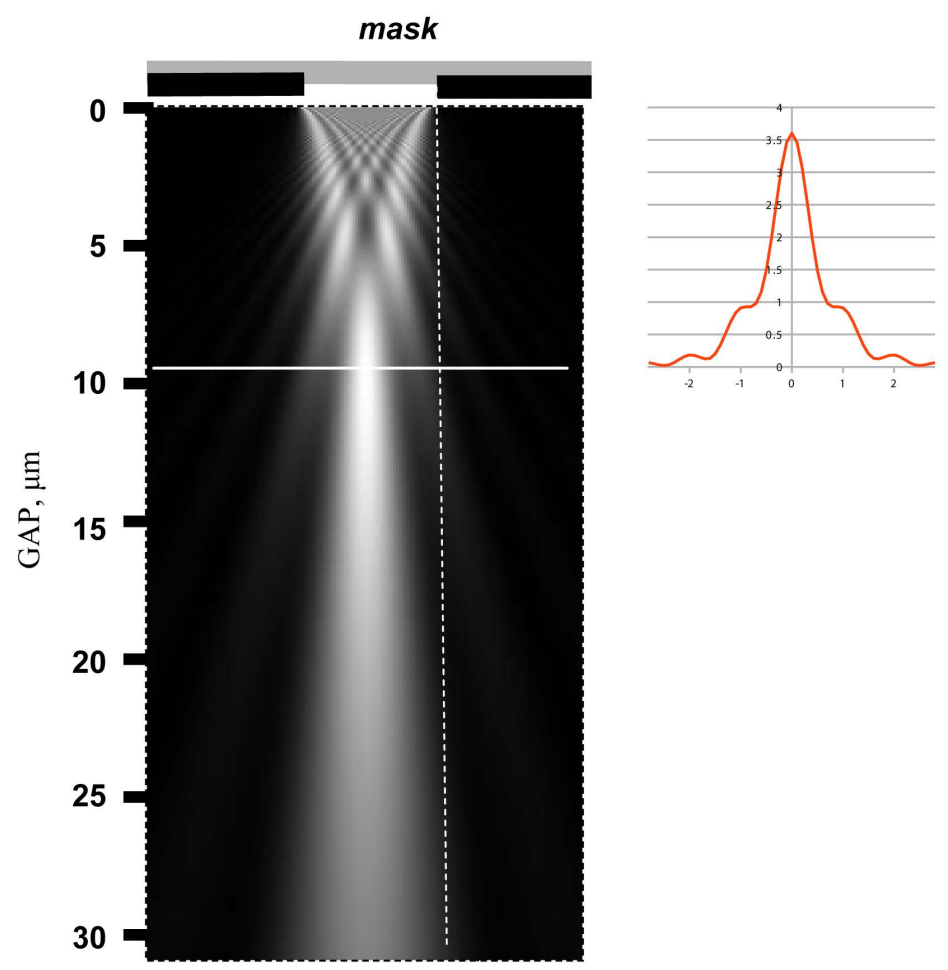

Figure 2. Simulated Fresnel pattern due to X-rays of wavelength $\lambda=0.8 \mathrm{~nm}$, focused from a slit of width $w=150 \mathrm{~nm}$ in cross-section. The inset at right shows the intensity profile at the Critical Gap $G_{c}=w^{2} / 3 \lambda$ [24]-[26], where the intensity of the incident plane wave was 2 . The abscissae are to scale and the slit width is 2.4 dimensionless units (see Ref. [19]). The dashed line includes, according to Heisenberg's uncertainty Principle, the effect on beam spread of the minimal transverse momentum introduced by the slit. The transverse momentum is greater in Fresnel diffraction where it is first convergent, and divergent after focus. Fresnel's method is more informative (see text).

This makes an interesting comparison with the experimentally verified Fresnel pattern shown in the Figure 2 in grayscale. At first sight, it seems - since the Fresnel diffraction focuses the slit so that the beam shrinks to one third of its width at the slit- -that the Uncertainty Principle is defied ${ }^{5}$. However closer inspection shows that the conflict can be re-construed. When the Fresnel image shrinks, the divergence angle increases to roughly three times the angle of the minimum uncertainty scattering. Then there is a crossover at focus and the beam passes from convergence to divergence, again at an angle greater than the minimum that transverse momentum requires. Beyond $60 \mu \mathrm{m}$ from the slit, the dashed line crosses the edge of the diverging Fresnel beam, so that diffraction continues to scatter with a transverse momentum greater than the minimum allowed by the Principle. The Fresnel beam in fact has an uncertainty closer to $p_{y}=\Delta p_{y}=-3 h / w$ (adjusting conveniently $0.881 \rightarrow 1$ for the rectangular transverse wave packet), consistent with the image width $\Delta y \simeq w / 3$. This illustrates an uncertainty in the definition of "position" in the Principle. According to the experimental data, the uncertainty of position could be roughly defined at the point of focus; not by the slit width! In summary: near field diffraction shows a beam width less than expected from Uncertainty based on the slit width. This is inconsistent with the limiting Principle; far field diffraction shows a momentum uncertainty that is larger than the slit width dictates, consistent with the limiting Principle—but systematically underestimated.

Moreover the focusing due to a top hat wave packet is yet more pronounced [19] [27]: the increased focusing induces increased uncertainty in the plane normal to the direction of propagation. The Principle therefore underestimates momentum uncertainty in the transverse plane due to diffraction from in-plane apertures, when focusing typically occurs. Of course, the image in Figure 2 does not represent a true stable wave packet because the transverse plane is restricted. This wave is more stable along the line of propagation, at least where $\lambda<w$.

${ }^{5}$ The convergent beam suggests that $p_{y}<0$; or that $\Delta p_{y}=-\Delta p_{y}$; or that the limiting sign in the Principle is reversible; or that the Principle lacks of definition. 


\section{Discussion}

The purpose of this discussion section is to provide perspective; not to repeat arguments already made. We set out originally to find the best wave function that would simulate the Uncertainty Principle. This turned out to be symmetric and Gaussian, so this was built into the wave function for a unidirectional plane wave. Because of minimal edge effects it was stable in the direction of propagation and approximately stable in the transverse plane.

The corresponding uncertainty is more precise than Heisenberg's Principle which in fact conflicts with established data. His Principle is confusingly and improperly defined. It needs supporting detail from wave mechanics.

Part of the detail is surprising: the carrier wave exists elastically in space beyond the speed of light, but without transporting energy. It provides an explanation for the reduction of the wave packet during quantum mechanical measurement.

There are other surprising consequences. Antiparticles occur as solutions to equations as they did for Dirac. The wave packet suggests conflicts in his solution and finds other wave mechanical solutions for particles and antiparticles.

Such a fundamental concept invites a new examination of modern science. After Newton, science was constructed as a deterministic theory; but modern quantum theory is imbedded with wave mechanics when in measurements on single particles, outcomes are typically unpredictable, as in diffraction. These outcomes are understood through probabilities and propensities. There are hidden variables [28] in the stable wave packet, but they are not sufficient to change the probable nature in individual measurement.

The uncertainty in a unidirectional wave packet is larger than in a two dimensional system such as a Bohr atom where the path is longer. The difference is a factor representing the ratio diameter/circumference, so that $\Delta p_{x} \cdot \Delta x \geq h / \pi$. In three dimensions, the apparent factor is again smaller by a factor about $\sqrt[3]{6}$. The combination is usually written $\Delta p_{x} \cdot \Delta x \geq h$.

\section{Conclusions}

Heisenberg's Uncertainty Principle conflicts with established experimental data and needs support from wave mechanics. This comes from the stable wave packet that is the principle vehicle for unidirectional photons or free particles with wide, planar, wave fronts. It is Gaussian, with minimal edge effects, and is the decay product of unstable packets. It is also well behaved, being differentiable and integrable; moreover it can be Fourier transformed and used with quantum operators. From this packet is derived a precisely defined uncertainty principle. The definition is fundamental and ideal; rather than over-simple and semi-empirical—perhaps even unexamined.

The packet differentiates, in wave mechanics, the transverse wave from its direction of propagation. In the transverse plane where the group velocity tends to zero, the phase velocity tends to infinity. Phase velocities beyond the horizon of the speed of light are used to explain the reduction of the wave packet for the case of Fresnel diffraction of a single photon. The packet thus contains hidden variables, but these are not sufficient to completely determine the reduction of the wave packet on detection of a single photon in a diffraction plane. In consequence, the site at which a single photon is absorbed must be treated with conventional probabilism. It remains in principle possible, by identifying hidden variables and excluding them experimentally, to recover determinism in physics. Most likely they would include phase information in both emitter and absorber. Whether or not determinism will ever be achieved, many physical and kinetic consequences follow the stable wave packet. Every photon that is ever measured is packaged [29].

\section{References}

[1] Popper, K.R. (1982) Quantum Theory and the Schism in Physics. Hutchison, 18.

[2] Dirac, P.A.M. (1958) The Principles of Quantum Mechanics. 4th Edition, OUP, Oxford, 121. http://dx.doi.org/10.1063/1.3062610

[3] Popper, K.R. (1982) Quantum Theory and the Schism in Physics. Hutchison, 22.

[4] Popper, K.R. (1982) Quantum Theory and the Schism in Physics. Hutchison, 10.

[5] Gell-Mann, M. (1979) In: Huff, D. and Prewett, O., Eds., The Nature of the Physical World, 1976 Nobel Conference, 
New York, 29.

[6] Bohm, D. and Bubb, J. (1966) Reviews of Modern Physics, 38, 453-475. http://dx.doi.org/10.1103/RevModPhys.38.453

[7] Popper, K.R. (1959) The Logic of Scientific Discovery. Hutchinson.

[8] Einstein, A., Podolski, B. and Rosen, N. (1935) Physical Review, 47, 777-780. http://dx.doi.org/10.1103/PhysRev.47.777

[9] Popper, K.R. (1982) Quantum Theory and the Schism in Physics. Hutchison, 15-22.

[10] Aspect, A. (2007) Nature, 446, 866-867. http://dx.doi.org/10.1038/446866a

[11] Aspect, A., Grangier, P. and Roger, G. (1982) Physical Review Letters, 49, 91-94. http://dx.doi.org/10.1103/PhysRevLett.49.91

[12] Aspect, A., Dalibard, J. and Roger, G. (1982) Physical Review Letters, 49, 1804-1807. http://dx.doi.org/10.1103/PhysRevLett.49.1804

[13] Longdell, J. (2011) Nature, 469, 475-476. http://dx.doi.org/10.1038/469475a

[14] Clausen, C., Usmani, I., Bussieres, F., Sangouard, N., Afzelius, M., de Riedmatten, H. and Gisin, N. (2011) Nature, 469, 508-511. http://dx.doi.org/10.1038/nature09662

[15] Saglamurek, E., Sinclair, N., Jin, J., Slater, J.A., Oblak, D., Bussieres, F., George, M., Ricken, R., Sohler, W. and Tittle, W. (2011) Nature, 469, 512-515. http://dx.doi.org/10.1038/nature09719

[16] Faipour, A., Xing, X. and Steinberg, A. (2011) Physical Review Letters, 107, Article ID: 133603.

[17] Von Neumann, J. (1932) Die Mathematischen Grundlagen der Quantenmechqanik.

[18] Bourdillon, A.J. (2015) Journal of Modern Physics, 6, 463-471. http://dx.doi.org/10.4236/jmp.2015.64050

[19] Jenkins, F.A. and White, H.E. (1981) Fundamentals of Optics. International Edition, McGraww-Hill, New York, 390.

[20] Dirac, P.A.M. (1958) The Principles of Quantum Mechanics. 4th Edition, Oxford University Press, Oxford, 261. http://dx.doi.org/10.1063/1.3062610

[21] Feynman, R.P. (1949) Physical Review, 76, 749-769. http://dx.doi.org/10.1103/PhysRev.76.749

[22] Stueckelberg, E.C.G. (1941) Helvetica Physica Acta, 14, 588-594.

[23] Littlefield, T.A. and Thorley, N. (1963) Atomic and Nuclear Physics and Introduction. Van Nostrand, 234.

[24] Bourdillon, A.J. and Vladimirsky, Y. (2006) X-Ray Lithography on the Sweet Spot.

[25] Bourdillon, A.J., Boothroyd, C.B., Kong, J.R. and Vladimirsky, Y. (2000) Journal of Physics D: Applied Physics, 33, 1-9. http://dx.doi.org/10.1088/0022-3727/33/17/307

[26] Jenkins, F.A. and White, H.E. (1981) Fundamentals of Optics. International Edition, McGraww-Hill, New York, 399.

[27] Bourdillon, A.J. and Boothroyd, C.B. (2005) Journal of Physics D: Applied Physics, 38, 2947-2951. http://dx.doi.org/10.1088/0022-3727/38/16/031

[28] Bell, J.S. (1966) Reviews of Modern Physics, 38, 447-452. http://dx.doi.org/10.1103/RevModPhys.38.447

[29] Bourdillon, A.J. Uncertainty and the Stable Wave Packet. www.Youtube.com/watch?v=A12c9uf75Hk 